\title{
A non-linear compartmental model to describe forage degradation kinetics during incubation in polyester bags in the rumen
}

\author{
BY M. S. DHANOA \\ Institute of Grassland and Environmental Research, Plas Gogerddan, Aberystwyth, \\ Dyfed SY23 3EB \\ AND J. FRANCE \\ Institute of Grassland and Environmental Research, North Wyke Research Station, \\ Okehampton, Devon EX20 2SB \\ AND R. C. SIDDONS \\ Formerly Institute of Grassland and Environmental Research, Hurley, Maidenhead, \\ Berkshire SL6 5LR \\ AND S. LOPEZ \\ Department of Animal Production, University of Leon, 24007 Leon, Spain \\ AND J. G. BUCHANAN-SMITH \\ Department of Animal and Poultry Science, University of Guelph, Guelph, Ontario N1G 2WI, \\ Canada
}

(Received 12 October 1993 - Revised 25 April 1994-Accepted 7 June 1994)

\begin{abstract}
A new model for describing forage degradation kinetics during incubation in the rumen using polyester bags is presented. Attention is given to dealing with the problem of deviations from exponential behaviour in the early stages of degradation by devising a function capable of representing exponential or sigmoidal trends. This is achieved by allowing part of the fractional degradation rate to vary with time of incubation, thus enabling responses other than those expected under simple first-order kinetics to be described. Seven sets of data consisting of 620 curves were analysed to study the performance of the new model compared with a commonly used exponential model. The proportion of significantly better fits varied from set to set. The new model deals successfully with sigmoidal behaviour and, thus, provides a means of analysing the degradation profiles of low-quality forage feeds.
\end{abstract}

Forage: Rumen: Compartmental model

The extent to which feed components, i.e. dry matter (DM), organic matter (OM), $\mathrm{N}$, etc. are degraded within the rumen is a function of both their rate of degradation and residence time. Rate of degradation has been studied using polyester bags containing feed samples placed in the rumen (e.g. Van Keuren \& Heinemann, 1962; Schoeman et al. 1972), and also by in vitro incubation of feed with either rumen microbes or enzymes (e.g. Broderick, 1978; Siddons et al. 1985b). Typically, the disappearance curves of DM, OM, neutral-detergent fibre (NDF), crude protein ( $N \times 6.25 ; \mathrm{CP}$ ) and other feed components obtained from these systems are then analysed using a model based on simple, first-order kinetics (Orskov \& 


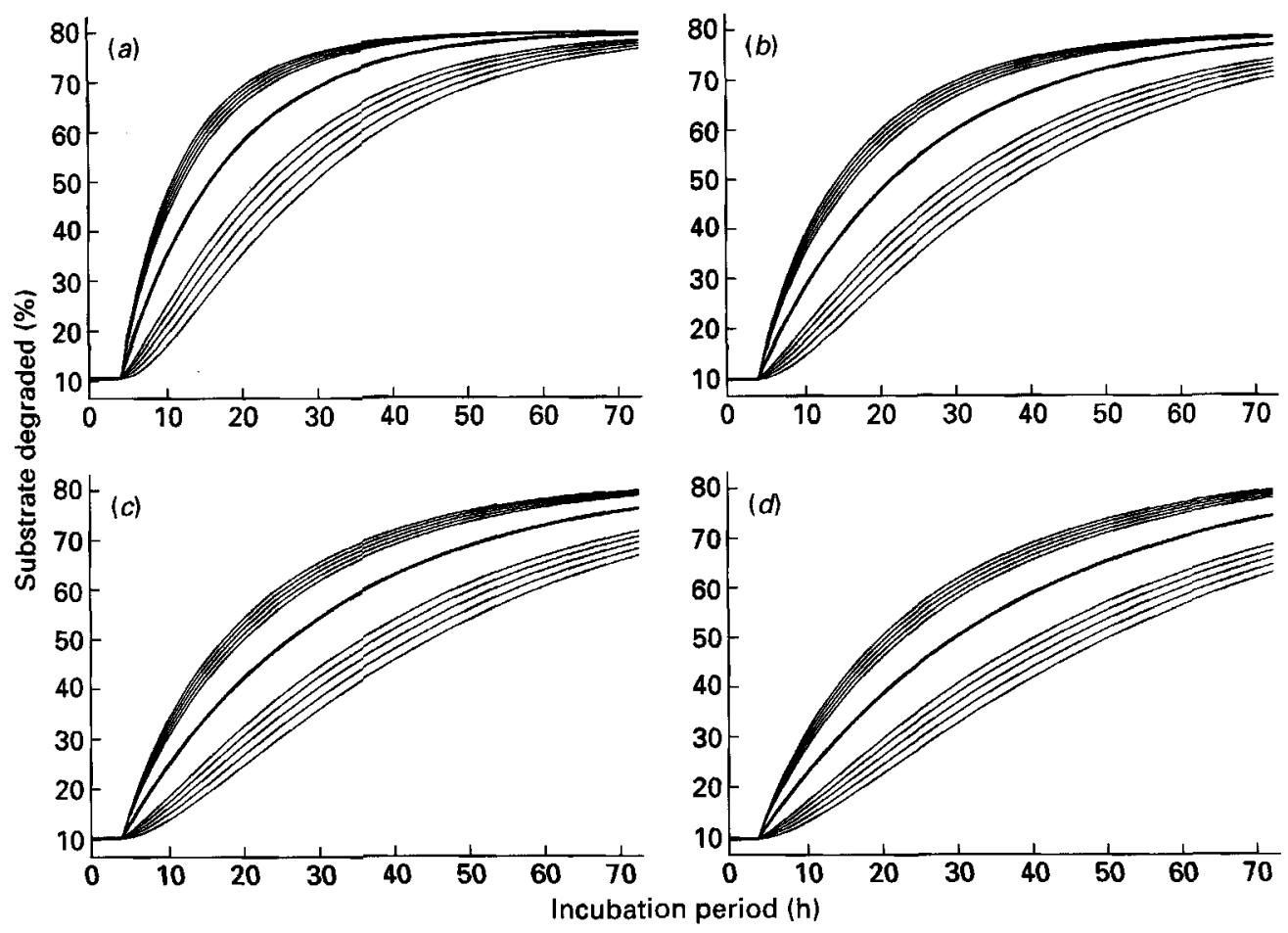

Fig. 1. Some shapes generated from equation 3 (see p. 5) by varying parameters $c$ and $d:(a) c=0 \cdot 0721,-0 \cdot 288$ $\leqslant d \leqslant+0.288,(b) c=0.0491,-0.196 \leqslant d \leqslant+0.196,(c) c=0.0368,-0.147 \leqslant d \leqslant+0.147,(d) c=0.03,-0.12$ $\leqslant d \leqslant+0 \cdot 12$. The middle curve is the simple exponential case $d=0$ and the curves above or below this correspond to positive or negative values of $d$ respectively. Parameters $a, b, T$ were assigned fixed values of $10,70,4$ respectively.

McDonald, 1979), with the apparent lag effect incorporated as suggested by McDonald (1981) and Dhanoa (1988). Mathematically the model may be summarized as follows:

$$
y=a+b\left(1-e^{-c(t-T)}\right), \quad t \geqslant T,
$$

where $y$ is disappearance ( $\mathrm{g}$ loss per $\mathrm{g}$ initially incubated), $t$ is time (h) and $a, b, c$, and $T$ are parameters. Parameter $a$ represents the soluble fraction (assumed instantly degradable), $b$ is the degradable part of the insoluble fraction, $c$ is the fractional degradation rate $(/ \mathrm{h})$ and $T$ is the lag time (h) before the commencement of degradation of $b$. Estimates of these parameters, used in conjunction with the estimate of ruminal rate of passage $k(/ \mathrm{h})$ obtained using digesta-flow markers and either faecal or rumen sampling, permit evaluation of the extent of ruminal degradation, $E$, by applying the formula:

$$
E=a+b c e^{-k T} /(c+k) \text {. }
$$

The term $e^{-k T}$ is the correction for the loss due to passage during the lag time.

Orskov \& McDonald (1979) used this model to investigate the degradation of soya-bean meal suspended in artificial-fibre bags in the rumen. The degradation of such protein supplements is essentially complete by $24 \mathrm{~h}$ of incubation and is generally satisfactorily described by assuming simple, first-order kinetics. However, with forages and forage-based diets much longer periods of incubation are normally required and analysis based on simple, first-order kinetics may not be appropriate. For example, time profiles on a semi- 
logarithmic scale of digestible residue remaining are not always linear as would be expected if a simple exponential is the true description of the profiles (see Table 1). The work of Harbers et al. (1981) also suggests that a constant fractional rate of degradation is not applicable to the latter stages of degradation of highly-lignified material. Hartley \& Dhanoa (1981) studied rates of degradation of plant cell walls and found that different sources of cell walls degraded at different rates. They showed that the kinetics of degradation were more complex than that represented by the simple exponential model.

Feed components such as DM, OM, CP and NDF are composed of a number of fractions, the amount and degradation rates of which are likely to differ. Consequently, even if the degradation of individual fractions obey first-order kinetics the overall rate of degradation would be expected to decline with time as the more-rapidly-degradable fractions are used up earlier. In the present paper a new model to describe forage degradation in the rumen is proposed and was derived by assuming the fractional rate of degradation may vary with time. Mathematically, the model can be considered a generalization of the simple exponential model and takes the form:

$$
y=a+b\left(1-e^{-c(t-T)-d(\sqrt{ } t-\sqrt{ } T)}\right),
$$

where $y, t, a, b$, and $T$ are defined as stated previously and $c$ and $d$ are parameters pertaining to the variable fractional rate of degradation (cf. equations 1 and 3 ). When $d=0$ the proposed new model (equation 3) is equivalent to the exponential model (equation 1). This new model was found to fit a wide range of incubation data on forages and forage-based diets successfully. This success is due to the many shapes which can be generated by altering the value of parameter $d$ for a given value of parameter $c$ (Fig. 1).

\section{MODEL DERIVATION}

Consider an amount (g) of a particular feed component entering the rumen and let the component consist of three fractions: (1) an instantly soluble fraction (or wash value), $W$, which is assumed to be degraded immediately; (2) an insoluble but potentially degradable fraction, $S$; and (3) an insoluble, undegradable fraction, $U$. Only $S$ and $U$ are of dynamic interest as $W$ is assumed degraded instantly. The scheme for the kinetics of the $S$ and $U$ pools is shown in Fig. 2, where $\mu$ and $k$ (both $/ \mathrm{h}$ ) denote the fractional rates of degradation and passage respectively. A discrete lag $T(\mathrm{~h})$ is assumed to occur before degradation commences and the fractional rate of degradation is postulated to vary with time as follows:

$$
\mu=c+d /(2 \sqrt{ } t), \quad t \geqslant T
$$

where $c(/ \mathrm{h})$ and $d\left(/ \mathrm{h}^{\frac{1}{2}}\right)$ are constants. The conditions $c \geqslant 0, d \geqslant-2 c \sqrt{ } T$ have to be satisfied as $\mu$ cannot be negative. Equation 4 permits the fractional degradation rate to remain constant, or decline or increase asymptotically with time. The fractional rate of passage $k$ is assumed constant and known. A value for $k$ can be obtained using digesta-flow markers and either rumen or faecal sampling (Siddons et al. 1985 ; France et al. 1985, 1988).

If the feed component is incubated in a polyester bag in the rumen or in a flask of rumen fluid in vitro, rather than placed directly in the rumen, then passage is zero and pool dynamics are described by the differential equations:

$$
\begin{gathered}
\mathrm{d} S / \mathrm{d} t=0, \quad 0<t<T, \\
=-\mu S, \quad t \geqslant T,
\end{gathered}
$$

and

$$
\mathrm{d} U / \mathrm{d} t=0, \quad t \geqslant 0
$$



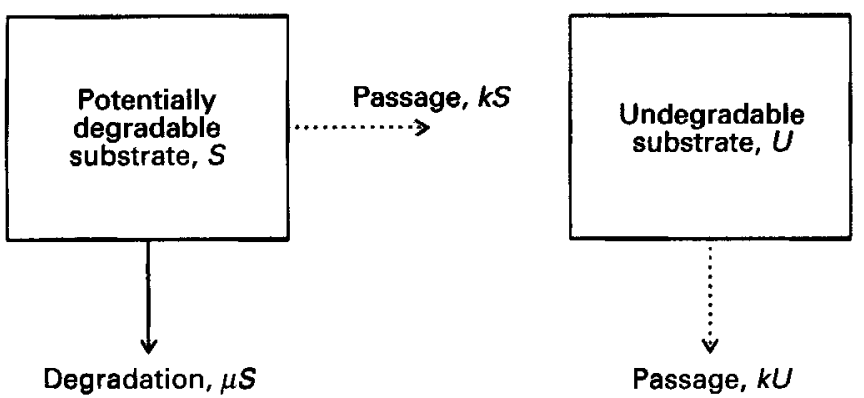

Fig. 2. Scheme for the kinetics of feed disappearance from the rumen. Removal of the passage fluxes $(\ldots \ldots \ldots$. gives the parallel scheme for disappearance from polyester bags in situ or from a flask in vitro.

On substituting for $\mu$ using equation 4 and integrating, equations 5 and 6 yield:

and

$$
\begin{gathered}
S=S_{0}, \quad 0<t<T, \\
=S_{0} e^{-[c(t-T)+d(\sqrt{ } t-\sqrt{ } T)]}, \quad t \geqslant T,
\end{gathered}
$$

where $S_{0}$ and $U_{0}$ are the zero-time quantities of the potentially-degradable and undegradable fractions of the feed component respectively. Cumulative degradation (i.e. disappearance) to time $t(\geqslant T)$ is given by:

$$
D=W+\int_{T}^{t} \mu S \mathrm{~d} t
$$

Substituting for $\mu$ and $S$ in this equation using equations 4 and $7 b$ respectively then integrating out yields:

$$
D=W+S_{0}\left\{1-e^{-[c(t-T)+d(\sqrt{ } t-\sqrt{ } T)}\right\} .
$$

Dividing equation 10 by the amount of feed component initially incubated (i.e. $W+S_{0}+U_{0}$ ) results in equation 3 .

If the feed component is placed in the rumen, rather than incubated in a bag or flask, then the principle of mass conservation gives the following differential equations:

and

$$
\begin{gathered}
\mathrm{d} S / \mathrm{d} t=-k S, \quad 0<t<T, \\
=-(\mu+k) S, \quad t \geqslant T,
\end{gathered}
$$

Their solution is:

$$
\mathrm{d} U / \mathrm{d} t=-k U, \quad t \geqslant 0 \text {. }
$$

and

$$
\begin{gathered}
S=S_{0} e^{-k t}, \quad 0<t<T, \\
=S_{0} e^{-[(c+k)(t-T)+d(\sqrt{ } t-\sqrt{ } T)+k T]}, \quad t \geqslant T,
\end{gathered}
$$

The extent of degradation in the rumen is given by the equation:

$$
E=\left(W+\int_{T}^{\infty} \mu S \mathrm{~d} t\right) /\left(W+S_{0}+U_{0}\right) .
$$

Using equations 4 and $13 b$ to substitute for $\mu$ and $S$ respectively in equation 15 , then integrating by parts yields:

$$
\begin{gathered}
E=\left[W+S_{0} e^{-k T}(1-k I)\right] /\left(W+S_{0}+U_{0}\right), \\
=a+b e^{-k T}(1-k I),
\end{gathered}
$$


where $a$ and $b$ are as defined earlier and:

$$
I=\int_{T}^{\infty} e^{-[(c+k)(t-T)+d(\sqrt{ } t-\sqrt{ } T)]} \mathrm{d} t .
$$

The integral $I$, which can be expressed in terms of the incomplete gamma function, is nonanalytical and, therefore, has to be evaluated numerically. The simple exponential model can be shown to be a special case of the new model by comparing equations 1 and 2 with equations 3 and $16 b$ respectively with $d$ set equal to zero.

\section{MATERIALS AND METHODS}

The data

Seven sets of data, yielding a total of 620 disappearance curves, were analysed. Set no. 1 (twenty-four curves) was obtained from a study undertaken on four sheep using two hays of three different lengths (large, medium and small) in which DM disappearance from polyester bags was recorded over incubation periods of up to $72 \mathrm{~h}$ for a medium-quality (approximately $50 \%$ DM digestibility (DMD)) perennial ryegrass (Lolium perenne) hay and $96 \mathrm{~h}$ for a lower-quality (approximately $40 \%$ DMD) mixed meadow hay (R. C. Siddons, unpublished results). Set no. 2 (twenty-eight curves) was obtained from a polyester-bag study on steers using four cuts (primary, mature, first and second regrowths) of S23 perennial ryegrass prepared in seven ways (as direct-cut silage, direct-cut ensiled with formic acid, wilted silage, wilted silage with formic acid, field-dried hay, direct-cut herbage, and wilted herbage) in which DM loss was monitored over $48 \mathrm{~h}$ (R. C. Siddons, unpublished results). Set no. 3 (forty-eight curves) came from a study on twelve Friesian steers using three cuts (primary growth, and mid- and late-season 4-week regrowths) of fresh herbage harvested daily from two pure swards (perennial ryegrass and white clover (Trifolium repens)). The disappearance of $\mathrm{OM}$ from polyester bags was recorded over incubation periods of up to $48 \mathrm{~h}$ (Beever et al. 1986). Set no. 4 (fifty-six curves) was obtained from a polyester-bag study undertaken on seven steers using brome (Bromis inermis) and lucerne (Medicago sativa) hay of two different lengths (large and small) in which DM disappearance of both hay and extrusa were monitored over $96 \mathrm{~h}$ (Dong, 1990). Set no. 5 (108 curves) came from a study on three sheep using hay from three botanical groups (grasses, legumes and weeds) cut in late June and early September. The disappearance of DM, OM, NDF, N, acid-detergent fibre and cellulose from polyester bags was recorded over incubation periods of up to $72 \mathrm{~h}$ (Lopez et al. 1991 a). Set no. 6 (seventytwo curves) came from a study on two sheep using three types of conserved forages (hay, fresh-freeze dried grass, and silage made in plastic bags) again cut in June and September. Disappearance of the same six feed components was monitored over $72 \mathrm{~h}$ (Lopez et al. 1991 b). Set no. 7 (260 curves) was taken from Mertens (1973), who reported results of NDF disappearance in vitro over $96 \mathrm{~h}$ for hays from a wide range of species and environmental growing conditions.

\section{Computational analysis}

For reasons of numerical efficiency, the proposed new model as given by equation 3 was transformed and fitted in the simpler form:

$$
y=A-B Q^{t} Z^{\sqrt{ } t},
$$

where $A, B, Z$ (all $>0$ ) and $Q(0<Q \leqslant 1)$ are parameters estimated by fitting equation 18 
to cumulative degradation data. Comparison of equation 18 with equation 3 yields the following relationships:

$$
\begin{aligned}
& c=-\ln Q, \\
& d=-\ln Z, \\
& b=A-a, \\
& T \ln Q+\sqrt{ } T \ln Z+\ln [B /(A-a)]=0 .
\end{aligned}
$$

Parameters $c, d, b$ and $T$ can thus, in theory, be calculated from estimates $A, B, Q$ and $Z$ if $a$, the instantly soluble fraction, is known. However, as cumulative degradation data from polyester-bag studies are generally positive, the fitted curve does not cross or touch the $t$-axis (the abscissa), and so equation 22 has no real roots. Therefore, we advocate the use of the minimum of the fitted function as an estimate of $T$, i.e.

$$
\sqrt{ } T=-\ln Z /(2 \ln Q) \text {. }
$$

The corresponding value of $y$ now provides an estimate of the parameter $a$.

Curve fitting was accomplished on a MicroVax 3600 mainframe computer using the maximum likelihood program (MLP; Ross, 1987), which permits direct iterative estimation of non-linear parameters using efficient algorithms such as Newton's method. The new model in the form of equation 18 conveniently separates into linear and non-linear components, and only a two-parameter space is needed for the non-linear iterative process. Initial parameter values are required to initiate this process, and the values adopted for $Q$ and $Z$ were 0.9 and 1.5 respectively. $Q$ and $Z$ were restored to these values at the start of each fit. The exponential model (equation 1) was fitted as described by Dhanoa (1988). Wash values (i.e. soluble fractions $a$ ) when available were not used in fitting either model on the grounds that they can exercise a disproportionate influence on the fitting process.

The success of any fit was judged on the basis of three criteria: (1) ability to describe the data without systematically over- or under-estimating any section of the curve, (2) biologically feasible parameter estimates, and (3) convergence to a solution in a small number of iterations. In the present study a solution was generally obtained ithin the default-set value of twenty iterations and its uniqueness was checked by perturbating the initial parameter values. Sensitivity to starting values, non-convergence, or convergence in a large number of iterations can be symptomatic of an ill-conditioned or inappropriate model.

MLP was also used for numerical integration. The AREA function, based on cubic interpolation, was employed to evaluate the integral $I$ (equation 17).

Residual variation was partitioned into three components using mean square prediction error (MSPE) analysis (Bibby \& Toutenburg, 1977):

$$
\begin{aligned}
& \text { MSPE }=\left[\Sigma(y-\hat{y})^{2}\right] / n, \\
& =(O-P)^{2}+s_{P}^{2}(1-m)^{2}+s_{o}^{2}\left(1-r^{2}\right),
\end{aligned}
$$

where $y$ and $\hat{y}$ denote the observed and predicted values respectively, $n$ is the number of observations, $O$ and $P$ are the respective means of the observed and predicted values, $s_{O}^{2}$ and $s_{P}^{2}$ are the respective variances, $m$ is the slope of regression line of $O$ on $P$ and $r$ is their correlation coefficient. Thus, MSPE can be partitioned into three components due to (1) the overall bias $(O-P)$ of prediction, (2) the deviation of the slope $m$ from line of unity and (3) the random variation around the regression line.

\section{RESULTS}

Semi-logarithmic plots of the seven data sets described earlier are summarized in Table 1, which suggests that the exponential (equation 1) can be an inappropriate choice of model. 
Table 1. Percentage of curves that are linear $(L)$ and quadratic $(Q)$ on a semi-logarithmic scale when using the undegraded residue remaining at 48, 72 and $96 \mathrm{~h}$ of incubation as the estimate of the undegradable fraction*

\begin{tabular}{|c|c|c|c|c|c|c|c|}
\hline \multirow[b]{3}{*}{ Data set no. $\dagger$} & \multirow{3}{*}{$\begin{array}{l}\text { No. of } \\
\text { curves }\end{array}$} & \multicolumn{6}{|c|}{ Incubation period $(\mathrm{h})$} \\
\hline & & \multicolumn{2}{|c|}{48} & \multicolumn{2}{|c|}{72} & \multicolumn{2}{|c|}{96} \\
\hline & & $\mathbf{L}$ & $\mathrm{Q}$ & $\mathbf{L}$ & $\mathrm{Q}$ & $\mathbf{L}$ & $Q$ \\
\hline 1 & 24 & 46 & 54 & 58 & 42 & 66 & 34 \\
\hline 2 . & 28 & 78 & 22 & - & - & - & - \\
\hline 3 & 72 & 82 & 18 & - & - & - & - \\
\hline 4 & 56 & 62 & 38 & 62 & 38 & 70 & 30 \\
\hline 5 & 108 & 86 & 14 & 92 & 8 & - & - \\
\hline 6 & 72 & 92 & 8 & 92 & 8 & - & - \\
\hline 7 & 260 & 75 & 25 & 86 & 14 & 84 & 16 \\
\hline
\end{tabular}

* For details of experimental procedures, see pp. 7-8.

+ For details, see p. 7.

Table 2. Comparison of the fit of the new model with that of the exponential using a partial $\mathrm{F}$ test to test the contribution of the extra parameter in the new model ${ }^{*}$

\begin{tabular}{cccc}
\hline & \multicolumn{3}{c}{ Significantly better fit (\%) at: } \\
\cline { 2 - 4 } Data set no. \begin{tabular}{ccc}
\hline \\
\cline { 2 - 4 }
\end{tabular} & $P \leqslant 0.20$ & $P \leqslant 0.10$ & $P \leqslant 0.05$ \\
\hline 1 & 45.8 & 33.3 & 29.2 \\
2 & 39.3 & 32.1 & 28.6 \\
3 & 26.4 & 13.9 & 8.3 \\
4 & 23.2 & 10.7 & 8.9 \\
5 & 38.9 & 30.6 & 19.4 \\
6 & 33.3 & 23.6 & 13.9 \\
7 & 58.5 & 41.2 & 31.5 \\
\hline
\end{tabular}

* For details of model, see p. 7.

$\dagger$ For details, see p. 7 .

The new model (equation 18) was fitted to the seven sets and, compared with the exponential, produced an improvement to each set, although the proportion of significantly better fits varied from set to set. Significance of the contribution of the extra parameter $Z$ was tested using a partial $F$ test. These results are summarized in Table 2 and confirm that all data profiles are not best described by the exponential, which being a robust model does fit and its inadequacies remain hidden. This is because the estimator of the rate-constant is easily found as the reciprocal of sample mean. Consequently, the exponential model can be fitted to curves which are the sum of two or more exponential components, to both concave and convex quadratic curves and to sigmoidal curves including cubic shapes, although in the case of symmetrical shapes the solution may converge to a straight line which is the limiting form of the exponential model.

The deficiencies of the exponential model in the analysis of forages are illustrated in Fig. $3(a)$, which shows a pattern of residuals (set no. 6) in the early stages of incubation that indicates its weakness in describing this stage adequately. The mathematical properties of 

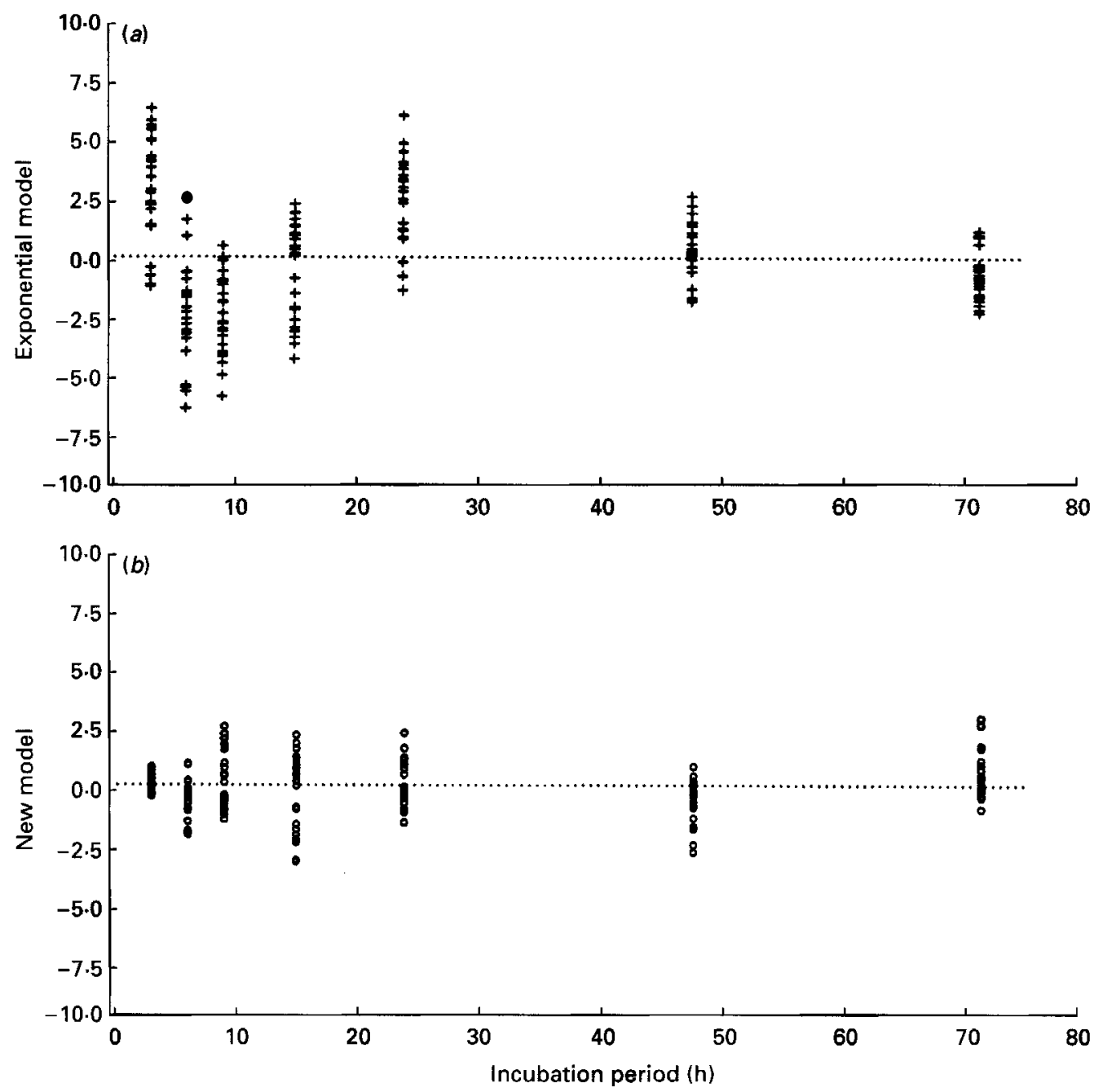

Fig. 3. (a) Typical pattern of residuals from fitting the simple exponential model showing systematic departures and $(b)$ reduction of such pattern when fitting the new model to the same data set. For details of models, see pp. $4-7$.

the exponential model are such that it cannot describe departures from diminishing returns behaviour. The new model was devised to deal with this aspect of degradation profiles and it is clear from Fig. 3(b) that it generally succeeds in doing so. Further evidence of this was obtained by calculating the number of runs of sign of the residuals, i.e. counting the number of sequences (one or more) of the same sign. It can be seen from Table 3 that the number of runs from fitting the exponential tends to be low, indicating systematic under or over fitting, whilst the number of runs from fitting the new model tends to be larger, indicating increased randomness of the residuals and the ability of the new model to describe the incubation profiles satisfactorily. The MSPE analysis identified the random component as accounting for most of the residual variation. However, total MSPE obtained by fitting the exponential was generally larger than that obtained by fitting the new model to the same incubation profile (Fig. 4). 
Table 3. Percentage of curves expressed according to number of runs of sign of the residuals from fitting the exponential (EXP) and new (MOD) models

(Smaller number of runs indicates systematic under- or over-fitting)

\begin{tabular}{|c|c|c|c|c|c|c|}
\hline \multirow[b]{2}{*}{ Data set no.* } & \multirow[b]{2}{*}{ Model† } & \multicolumn{5}{|c|}{ No of runs: } \\
\hline & & $\leqslant 4$ & 5 & 6 & 7 & $>7$ \\
\hline 1 & $\begin{array}{l}\text { EXP } \\
\text { MOD }\end{array}$ & $\begin{array}{c}41 \cdot 7 \\
0\end{array}$ & $\begin{array}{l}16 \cdot 7 \\
25 \cdot 0\end{array}$ & $\begin{array}{l}33 \cdot 3 \\
25 \cdot 5\end{array}$ & $\begin{array}{r}8 \cdot 3 \\
20 \cdot 8\end{array}$ & $\begin{array}{l}0 \\
8 \cdot 3\end{array}$ \\
\hline 2 & $\begin{array}{l}\text { EXP } \\
\text { MOD }\end{array}$ & $\begin{array}{l}0 \\
0\end{array}$ & $\begin{array}{l}0 \\
0\end{array}$ & $\begin{array}{r}10 \cdot 7 \\
3.6\end{array}$ & $\begin{array}{l}14 \cdot 3 \\
17 \cdot 9\end{array}$ & $\begin{array}{l}75 \cdot 0 \\
78 \cdot 5\end{array}$ \\
\hline 3 & $\begin{array}{l}\text { EXP } \\
\text { MOD }\end{array}$ & $\begin{array}{r}58 \cdot 3 \\
4 \cdot 2\end{array}$ & $\begin{array}{l}30-6 \\
62 \cdot 5\end{array}$ & $\begin{array}{l}12 \cdot 5 \\
31.9\end{array}$ & $\begin{array}{l}0 \\
0\end{array}$ & $\begin{array}{l}0 \\
0\end{array}$ \\
\hline 4 & $\begin{array}{l}\text { EXP } \\
\text { MOD }\end{array}$ & $\begin{array}{l}7 \cdot 1 \\
0\end{array}$ & $\begin{array}{r}17 \cdot 9 \\
8 \cdot 9\end{array}$ & $\begin{array}{l}26 \cdot 8 \\
19 \cdot 6\end{array}$ & $\begin{array}{l}25 \cdot 0 \\
17 \cdot 9\end{array}$ & $\begin{array}{l}23 \cdot 2 \\
53 \cdot 6\end{array}$ \\
\hline 5 & $\begin{array}{l}\text { EXP } \\
\text { MOD }\end{array}$ & $\begin{array}{r}44.4 \\
1.9\end{array}$ & $\begin{array}{l}16 \cdot 7 \\
47 \cdot 2\end{array}$ & $\begin{array}{l}38 \cdot 0 \\
42 \cdot 6\end{array}$ & $\begin{array}{r}0 \cdot 9 \\
10 \cdot 2\end{array}$ & $\begin{array}{l}0 \\
0\end{array}$ \\
\hline 6 & $\begin{array}{l}\text { EXP } \\
\text { MOD }\end{array}$ & $\begin{array}{c}37 \cdot 5 \\
0\end{array}$ & $\begin{array}{l}36 \cdot 1 \\
63 \cdot 9\end{array}$ & $\begin{array}{l}19 \cdot 4 \\
19 \cdot 4\end{array}$ & $\begin{array}{r}6 \cdot 9 \\
16 \cdot 7\end{array}$ & $\begin{array}{l}0 \\
0\end{array}$ \\
\hline 7 & $\begin{array}{l}\text { EXP } \\
\text { MOD }\end{array}$ & $\begin{array}{c}23.5 \\
0\end{array}$ & $\begin{array}{l}52 \cdot 7 \\
71 \cdot 1\end{array}$ & $\begin{array}{l}15 \cdot 0 \\
11 \cdot 2\end{array}$ & $\begin{array}{r}6.5 \\
15.0\end{array}$ & $\begin{array}{l}2 \cdot 3 \\
2 \cdot 7\end{array}$ \\
\hline
\end{tabular}

* For details, see p. 7.

$\uparrow$ For details, see pp. 4-7.

The values of extent of degradation calculated following fitting the new and exponential models are illustrated in Table 4 using two of the data sets (no. 2 and no. 4). Extent was determined using equation 2 for the exponential and equations $16 b$ and 17 for the new model and assuming a passage rate of $002 / \mathrm{h}$. Wash values were available for both data sets and were used as estimates of the soluble fractions $a$ in this comparison. Table 4 shows that the choice of model has some bearing on the estimate of extent of degradation, although the differences for the two sets of data are perhaps too small to lead to any firm conclusions. Because the influence of a model is limited to the description of the insoluble fraction only, Table 4 also contains values of extent excluding the soluble fraction (wash value), which is assumed totally and instantly degradable.

To illustrate the effect of model on the extent of degradation, the estimate obtained using the new model was expressed as a proportion of the corresponding estimate obtained using the exponential model, i.e. the pair-wise extent ratio (PER). Values of PER greater than unity indicate higher estimates of extent given by the new model and values less than unity a lower estimate. The proportion of cases exhibiting 2 or $5 \%$ overestimates (i.e. PER = 1.02 or 1.05 ) and underestimates (PER $=0.98$ or 0.95 ) for data sets no. 2 and no. 4 considering all cases or just those better fitted by the new model and with the wash value included or excluded are shown in Table 5. For both data sets, excluding the wash value tended to increase the differences between the models. For data set no. 2, using only those cases which were better fitted by the new model had little effect on the proportion of overand underestimates, whereas for data set no. 4 the majority of the better-fitted curves exhibited a lower estimate of extent of degradation with the new model. 


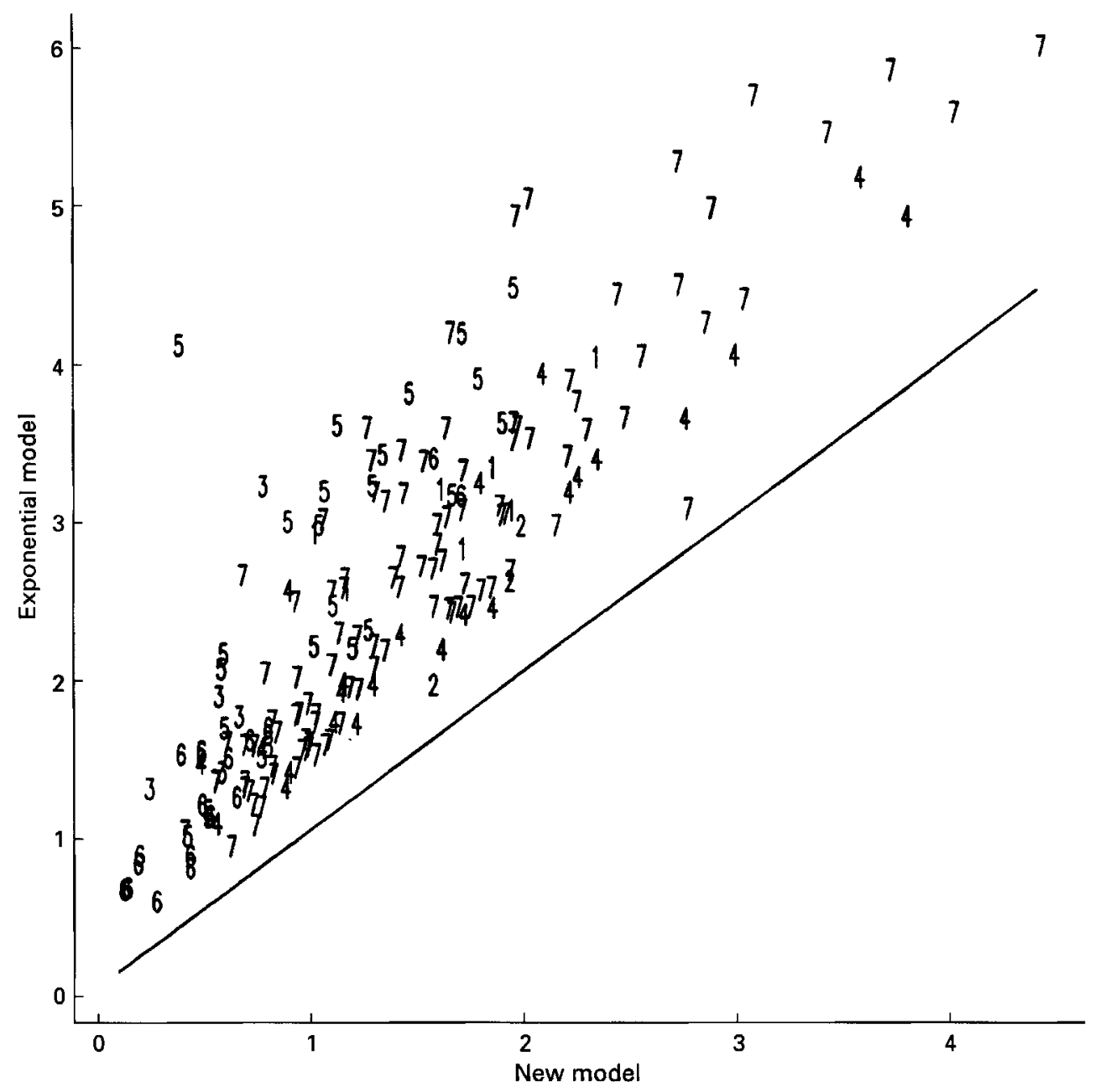

Fig. 4. Root mean square prediction error (\%) calculated from the fit of the simple exponential model and from corresponding fit of the new model to the same incubation profile. The numbers identify profiles from each of the seven data sets and the line of unity indicates no difference. Only those profiles which were significantly better described by the new model are indicated. For details of models and procedures, see pp. 4-7.

\section{DISCUSSION}

Knowledge of degradation kinetics is important because they define or determine the proportion of consumed nutrients that is utilized by the animal. To study in vivo degradation kinetics specific to a feed, usually polyester bags containing a sample of that feed are placed in the rumen of fistulated animals. Before degradation can commence the processes of hydration, attachment and colonization of the insoluble substrate by the rumen microbes are initiated. A many-fold increase in the size of the associated microbial population leads to a phase of rapid degradation of part of the substrate during which more-readily-degradable fractions are exhausted. The remaining substrate then is degraded at a slower rate until all the residue consists of undegradable fraction only. The combined effect of these three stages gives rise to degradation profiles which are sigmoidal to varying degrees. Also the heterogeneous nature of feed components such as DM, OM, CP and 
Table 4. Extent of degradation calculated using estimates of the parameters obtained by

fitting the exponential $(E X P)$ and new $(M O D)$ models

(Calculations assume a passage rate value of $0.02 / \mathrm{h}$. Only those profiles which were significantly better described by the new model are included in the comparison)

\begin{tabular}{|c|c|c|c|c|c|}
\hline \multirow[b]{2}{*}{ Wash value } & \multirow[b]{2}{*}{ Data set no.* } & \multirow[b]{2}{*}{ Model $\uparrow$} & \multicolumn{3}{|c|}{ Extent of degradation $(\%)$} \\
\hline & & & Minimum & Mean & Maximum \\
\hline Included & $\begin{array}{l}2 \\
4\end{array}$ & $\begin{array}{l}\text { EXP } \\
\text { MOD } \\
\text { EXP } \\
\text { MOD }\end{array}$ & $\begin{array}{l}61 \cdot 9 \\
70 \cdot 5 \\
40 \cdot 5 \\
37 \cdot 5\end{array}$ & $\begin{array}{l}77 \cdot 4 \\
78 \cdot 6 \\
53 \cdot 3 \\
50 \cdot 6\end{array}$ & $\begin{array}{l}85 \cdot 0 \\
83 \cdot 5 \\
65 \cdot 4 \\
65 \cdot 2\end{array}$ \\
\hline Excluded & $\begin{array}{l}2 \\
4\end{array}$ & $\begin{array}{l}\text { EXP } \\
\text { MOD } \\
\text { EXP } \\
\text { MOD }\end{array}$ & $\begin{array}{l}22 \cdot 6 \\
23 \cdot 9 \\
29 \cdot 7 \\
27 \cdot 0\end{array}$ & $\begin{array}{l}36 \cdot 6 \\
37 \cdot 8 \\
35 \cdot 5 \\
32 \cdot 8\end{array}$ & $\begin{array}{l}56 \cdot 5 \\
53 \cdot 3 \\
41 \cdot 2 \\
38.9\end{array}$ \\
\hline
\end{tabular}

* For details, see p. 7.

$\dagger$ For details, see pp. 4-7.

Table 5. Proportion of cases exhibiting a pair-wise extent ratio greater than 1.02 and 1.05 and less than 0.98 and 0.95 , i.e. when extent of degradation obtained using the new model was compared with that obtained using the exponential model*

\begin{tabular}{|c|c|c|c|c|c|c|}
\hline \multirow[b]{2}{*}{ Data set no. $\dagger$} & \multirow[b]{2}{*}{ No. of curves } & \multirow[b]{2}{*}{ Wash value } & \multicolumn{4}{|c|}{ Pair-wise extent ratio } \\
\hline & & & $\geqslant 1.05$ & $\geqslant 1.02$ & $\leqslant 0.98$ & $\leqslant 0.95$ \\
\hline 2 & $\begin{array}{l}28 \ddagger \\
11 \S\end{array}$ & $\begin{array}{l}+ \\
- \\
+ \\
-\end{array}$ & $\begin{array}{l}0.14 \\
0.32 \\
0.18 \\
0.27\end{array}$ & $\begin{array}{l}0.29 \\
0.46 \\
0.18 \\
0.46\end{array}$ & $\begin{array}{l}0.25 \\
0.36 \\
0.27 \\
0.46\end{array}$ & $\begin{array}{l}0.00 \\
0 \cdot 18 \\
0 \cdot 00 \\
0.27\end{array}$ \\
\hline 4 & $\begin{array}{l}56 \ddagger \\
14 \$\end{array}$ & $\begin{array}{l}+ \\
- \\
+ \\
-\end{array}$ & $\begin{array}{l}0.11 \\
0.23 \\
0.00 \\
0.00\end{array}$ & $\begin{array}{l}0.25 \\
0 \cdot 32 \\
0.00 \\
0.00\end{array}$ & $\begin{array}{l}0.34 \\
0.45 \\
0.71 \\
0.79\end{array}$ & $\begin{array}{l}0.21 \\
0.27 \\
0.64 \\
0.71\end{array}$ \\
\hline
\end{tabular}

* For details, see pp. 4-7.

$\dagger$ For details, see p. 7.

$\ddagger$ All curves.

$\S$ Only those curves better fitted by the new model.

NDF with different associated degradation kinetic characteristics adds to the complexity of overall kinetics. On these and other accounts such as microbial biomass contamination, it is biologically unlikely that overall kinetics will be simple first-order kinetics. Semilogarithmic plots of (degradable) substrate remaining displaying non-linearity in the early stage of incubation are evidence of this (Table 1).

On a theoretical basis, therefore, initially microbial growth during colonization might be expected to be associated with an increasing rate of substrate degradation, whereas subsequently the rate of degradation might fall due to an inhibitory effect on microbial activity of an increase in the proportion of more-lignified less-readily-degradable substrate. The possible acceleration of substrate degradation due to an increase in microbial activity and inhibition effects of undegradable substrate accumulation, however, have received little 
attention in the literature. Recently France et al. (1990) have addressed these aspects of degradation and found that inhibition is best modelled implicitly using a negative exponential function of time, whilst acceleration of degradation due to microbial activity can be represented explicitly, although this requires certain restrictive assumptions (e.g. constant rumen volume). Nevertheless, some useful sigmoidal-type models have emerged which provide alternatives in analysing polyester-bag data for forages. Some of the new models challenge our concepts of forage degradation and it is hoped that further improvements and refinements will emerge.

These models, although theoretically attractive to explore and understand degradation kinetics, are less suitable for routine use because they are not sufficiently robust to deal with relatively noisy experimental data. In order to produce a utilitarian model, it was decided to express the fractional degradation rate as a simple function of time $t$, i.e. $\mu=c+\mathrm{d} t^{\alpha}$ where $\alpha$ could have a range of values. However, it was found that keeping $\alpha$ as an extra parameter rendered the model ill-conditioned and the mathematics intractable. From a range of values of $\alpha$ such that $\mu$ will tend to an asymptote for large values of $t$ it was found that $\alpha=-\frac{1}{2}$ was suitable and led to the present model, which successfully described various data sets. The fractional rate $\mu$ monotonically tends to the value of $c$ as $t$ increases. The model reduces to the exponential model when $d=0$. The generalization adopted makes the model quite versatile and it copes well with changing shapes of degradation profiles. This is evident from the results summarized in Tables 2 and 3 . The new model fitted significantly better in a large proportion of cases (Table 2) and, thus, dictates that the exponential be used judiciously. The 'runs test' applied to the residuals from the fit of these models showed an increase in the number of runs with the new model, thus reducing systematic bias and better mimicking the degradation profiles.

From the present study it is clear that there are important deviations from simple firstorder kinetics when analysing degradation profile data from forages, forage-based and other low-quality feeds. This is apparent from the good description of such data by the new model. Clearly there is a need for caution when using simple first-order models. Discrete lag models have been used as an approximation of both lag and sigmoidal behaviour but they are difficult to justify biologically. The new model is capable of describing lag and sigmoidal behaviour separately, thus providing a means of analysis of degradation profiles of low-quality feeds $(<40 \%$ DMD).

\section{REFERENCES}

Broderick, G. A. (1978). In vitro procedures for estimating rates of ruminal protein degradation and proportions of protein escaping the rumen undegraded. Journal of Nutrition 108, 181-190.

Beever, D. E., Dhanoa, M. S., Losada, H. R., Evans, R. T., Cammell, S. B. \& France, J. (1986). The effect of forage species and stage of harvest on the processes of digestion occurring in the rumen of cattle. British Journal of Nutrition 56, $439-454$.

Bibby, J. \& Toutenberg, H. (1977). Prediction and Improved Estimation in Linear Models. London: John Wiley $\&$ Sons.

Dhanoa, M. S. (1988). On the analysis of dacron bag data for low degradability feeds. Grass and Forage Science 43, $441-444$.

Dong, Y. (1990). The significance of chewing during eating and rumination on forage digestion in cattle. MSc Thesis, University of Guelph.

France, J., Dhanoa, M. S., Siddons, R. C., Thornley, J. H. M. \& Poppi, D. P. (1988). Estimating the production of faeces by ruminants from faecal marker concentration curve. Journal of Theoretical Biology 135, 383-391.

France, J., Thornley, J. H. M., Dhanoa, M. S. \& Siddons, R. C. (1985). On the mathematics of digesta flow kinetics. Journal of Theoretical Biology 113, 743-758.

France, J., Thornley, J. H. M., Lopez, S., Siddons, R. C., Dhanoa, M. S., Van Soest, P. J. \& Gill, M. (1990). On the two compartment model for estimating the rate and extent of feed degradation in the rumen. Journal of Theoretical Biology 146, 269-287.

Harbers, L. H., Brazle, F. K., Raiten, D. J. \& Owensby, C. E. (1981). Microbial degradation of smooth brome and tall fescue observed by scanning electron microscopy. Journal of Animal Science 51, 439-446. 
Hartley, R. D. \& Dhanoa, M. S. (1981). Rates of degradation of plant cell walls measured with a commercial cellulase preparation. Journal of Science of Food and Agriculture 32, 849-856.

Lopez, S., Carro, M. D., Gonzalez, J. S. \& Ovejero, F. J. (1991 a). Rumen degradation of the main forage species harvested from permanent mountain meadows in North-western Spain. Journal of Agricultural Science, Cambridge 117, 363-369.

Lopez, S., Carro, M. D., Gonzalez, J. S. \& Ovejero, F. J. (1991b). The effect of method of forage conservation and harvest season on the degradation of forages harvested from permanent mountain meadows. Animal Production 53, 177-182.

McDonald, I. (1981). A revised model for the estimation of protein degradability in the rumen. Journal of Agricultural Science, Cambridge 96, 251-252.

Mertens, D. R. (1973). Application of mathematical models to cell wall digestion and forage intake in ruminants, $\mathrm{PhD}$ Thesis, Cornell University.

Ørskov, E. R. \& McDonald, I. (1979). The estimation of protein degradability in the rumen from incubation measurements weighted according to rates of passage. Journal of Agricultural Science, Cambridge 92, 449-503.

Ross, G. J. S. (1987). MLP, Maximum Likelihood Program, version 3.08. Oxford: Numerical Algorithms Group.

Schoeman, E. A., De Vet, P. J. \& Burger, W. J. (1972). The evaluation of the digestibility of treated proteins. Agroanimalia 4, 35-46.

Siddons, R. C., Paradine, J., Beever, D. E. \& Cornell, P. R. (1985a). Ytterbium acetate as a particulate-phase digesta flow marker. British Journal of Nutrition 54, 509-519.

Siddons, R. C., Paradine, J., Gale, D. L. \& Evans, R. T. (1985 b). Estimation of the degradability of dietary protein in the sheep rumen by in vivo and in vitro procedures. British Journal of Nutrition 54, 545-561.

Van Keuren, R. W. \& Heinemann, W. W. (1962). Study of a nylon bag technique for in vivo estimation of forage digestibility. Journal of Animal Science 21, 340-345. 